Journal of Mathematical Biology manuscript No.

(will be inserted by the editor)

C. J. W. Breward · H. M. Byrne · C. E. Lewis

\title{
The role of cell-cell interactions in a two-phase model for avascular tumour growth
}

\author{
3rd May 2001
}

\begin{abstract}
A two-phase model is presented to describe avascular tumour growth. Conservation of mass equations, including oxygen-dependent cell growth and death terms, are coupled with equations of momentum conservation. The cellular phase behaves as a viscous liquid, while the viscosity of the extracellular water manifests itself as an interphase drag. It is assumed that the cells become mechanically stressed if they are too densely packed and that the tumour will try to increase its volume in order to relieve such stress. By contrast, the overlapping filopodia of sparsely populated cells create short-range attractive effects. Finally, oxygen is consumed by the cells as it diffuses through the tumour.

The resulting system of equations are reduced to three, which describe the evolution of the tumour cell volume fraction, the cell speed and the oxygen tension. Numerical simulations indicate that the tumour either evolves to a travelling wave profile, in which it expands at a constant rate, or it settles to a steady state, in which the net rates of cell proliferation and death balance. The impact of varying key model parameters such as cellular viscosity, interphase drag, and cellular tension are discussed. For example, tumours consisting of well-differentiated (i.e. viscous) cells are shown to grow more slowly than those consisting of poorly-differentiated (i.e. less viscous) cells. Analytical results for the case of oxygen-independent growth are also presented, and the effects of varying the key parameters determined (the results are in line with the numerical simulations of the full problem). The key results and their biological implications are then summarised and future model refinements discussed.
\end{abstract}

\section{Introduction}

The avascular stage of tumour growth is characterised by small tumours which gain the nutrients and oxygen they need for survival and growth by diffusion in from external blood vessels. Since there are no blood vessels within the tumour to supply the mass needed for such volume expansion, this must also enter through the tumour's periphery.

Avascular tumour growth has been widely studied using mathematical techniques, and the resulting models are becoming increasingly sophisticated. For example, in an early paper [16], Greenspan presented a compartmentalised model in which the tumour was divided into proliferating and quiescent regions. Recently, Ward and King incorporated more realistic functional forms for the cell birth and death rates, which depend on the local oxygen tension in the tumour tissue [28,29]. They also distinguished between live and dead cell populations. A common feature of the models presented in $[17,28,29]$ is that low oxygen tensions are responsible for generating substantial cell death, and for the resulting formation of areas of necrosis within the tumours. Landman and Please [22] propose an alternative mechanism for the formation of regions of necrosis. They view the tumour as a two-phase mixture of cells and water, each phase having a different pressure. In viable regions of the tumour, where cells proliferate, the cell volume fraction is taken to be constant and the cell pressure is assumed to exceed the water pressure - the cells are compacted and pressure is transmitted from cell-to-cell. They assume that "necrotic" regions form when the pressures in the two phases become equal and the cells are no longer compacted. This hypothesis has yet to be demonstrated experimentally.

C. J. W. Breward e-mail: breward@maths.ox.ac.uk, C. E. Lewis: Academic Unit of Pathology, Section of Pathology and Oncology, University of Sheffield Medical School, Beech Hill Road, Sheffield. S10 2RX. UK

C. J. W. Breward, H. M. Byrne: Centre for Mathematical Medicine, Division of Theoretical Mechanics, School of Mathematical Sciences, University of Nottingham, University Park, Nottingham. NG7 2RD. UK

C. J. W. Breward: Current Address: Oxford Centre for Industrial and Applied Mathematics, Mathematical Institute, 24-29 St Giles, Oxford. OX1 3LB

Key words: Two-phase modelling; tumour growth; avascular; cellular viscosity; cell-cell stresses; numerical solution 
De Angelis and Preziosi [10] relax the assumption that the cell volume fraction in the viable region of the tumour be constant and develop an alternative approach in which cells are assumed to attract each other when the cell volume fraction is below a threshold value and to repel each other when this value is exceeded.

In this paper we generate a new mathematical model for the development of an avascular tumour which incorporates a number of the phenomena introduced in the papers described above. As in [22], we view the tumour as a two-phase material, consisting of tumour cells and extracellular water. Note that there are other phases present in avasular tumours; we discuss the formation of three-phase models which incorporate, for example, extracellular matrix, in the discussion. We also assume that necrosis is initiated by low oxygen tensions, following $[28,29]$. We assume that there is internal conservation of mass, and that changes in tumour volume are due to the in-flow of liquid across the tumour boundary: this fluid is taken up by cells which are growing and undergoing mitosis. We suppose that the behaviour of the cellular phase is similar to that of a viscous fluid, the viscosity indicating the degree of differentiation of the tumour cells: poorly differentiated tumours (i.e. the most aggressive tumours) are characterised by less intercellular cohesion and are thus "less viscous" than their well-differentiated counterparts, see [13,23].

Our final key assumption is that the pressure in the cellular phase differs from that in the extracellular water phase due to interactions between the cells. Rather than fixing the cell volume fraction, we adopt a similar approach to $[6,7,10]$ and allow cells to attract or repel each other, depending on their local volume fraction. We associate cellular attraction with overlapping filopodia, and cellular repulsion with the relief of mechanical stress caused by deformation of the cellular membrane.

Our model has its foundations in two-phase flow models, and, as such, follows the approach taken by Fitt et al. [12]. However, our model allows for matter to change phase due to cell birth and death and, as such, differs from the numerous two-phase models that are used to describe nonbiological applications, in which there is no internal phase generation. For simplicity, we formulate our model in a one-dimensional (cartesian) geometry. All the equations and boundary conditions in our model could be easily replaced with their radially-symmetric or fully three dimensional counterparts. We expect that the results obtained from analysing our one-dimensional model would be qualitatively similar to those obtained using a more complicated geometry. We formulate conservation of mass and momentum equations for the two phases, and a diffusion-consumption equation to describe the oxygen tension within the tumour mass (we focus on the oxygen tension rather than any other nutrients, assuming that it is the single, rate-limiting nutrient within the tumour volume). We close the model equations by specifying appropriate constitutive laws. The first law states that there are no voids within the tumour, and the second describes the way in which cell-cell interactions influence the pressure in the cell phase.

Our model provides us with predictions for the effects of varying the cellular viscosity $(i . e$. the differentiation status of the tumour cells), the interphase drag (which we correlate with the viscosity of the extracellular water) and the membrane tension. We are also able to justify the use of diffusion-like equations describing tumour growth: our cell-cell interactions manifest themselves as a nonlinear diffusivity, in the limit as viscous effects are neglected. The modelling strategy described in this paper also provides a framework for easily incorporating stress-dependent cell-proliferation and death terms. All these insights are discussed in the text or in section 6 .

Throughout this paper we avoid identifying the actual magnitude of the physical parameters. This is because we are interested in generating a generic model that can then be used (in various subforms) to describe the growth of a wide variety of tumour cell lines. Also, to our knowledge, no complete set of data exists for any specific cell line, and we believe that it would be inconsistent to quote an incomplete set of data.

Our underlying aim for generating the model presented in this paper is to use it as a building block for producing a model that describes vascular tumour growth, that is, the late stage of growth in which the tumour has aquired its own blood supply. Such models will be useful for studying vascular tumour growth both at the microscale and at the macroscale. At the microscale, we are concerned with interactions between a single, compliant blood vessel and the tumour cells that it supplies with nutrients (see [2,21]); at the macroscale, we are concerned with predicting how the tumour as a whole grows (see $[4,18]$ ).

In section 2 we formulate our mathematical model, which describes the evolution of the tumour. In section 3 we explain how the model may be simplified and nondimensionalise the governing equations, before discussing the behaviour of the tumour near its outer boundary. In section 4 we present numerical simulations of the model equations for several parameter regimes of physical interest. We analyse the initial 
growth of the tumour in section 5.1 before considering whether the tumour evolves to a steady state in section 5.2. We analyse the model for the case in which oxygen levels are uniformly high within the tumour in section 5.3. Finally we present our conclusions and a discussion in section 6 .

\section{Mathematical model}

We denote the volume fractions of tumour cells and extracellular water by $\alpha$ and $\beta$. We formulate conservation of mass equations under the assumption that each phase has the same density. This is a reasonable assumption in a two-phase model, since we assume that cells are built from the extracellular water, and that the products of cell death are extracellular water. We denote by $x$ and $t$ the independent variables and write

$$
\frac{\partial \alpha}{\partial t}+\frac{\partial}{\partial x}\left(u_{c} \alpha\right)=q_{c} \quad \text { and } \quad \frac{\partial \beta}{\partial t}+\frac{\partial}{\partial x}\left(u_{w} \beta\right)=q_{w} .
$$

In equations (1), $u_{c}$ is the speed with which the tumour cells move, $u_{w}$ the speed with which the water phase moves, $q_{c}$ represents the rate of change of tumour cell volume due to cell birth and death, and $q_{w}$ represents the complementary rate of change of extracellular water volume due to cell birth and death. We assume that there are no voids and so we set

$$
\alpha+\beta=1 .
$$

We further assume that momentum is conserved. Denoting the stresses in the cellular and extracellular phases by $\sigma_{c}$ and $\sigma_{w}$, and neglecting inertia (we may check, a posteriori, that the Reynolds number for the flow, $R e=\rho L U / \mu_{c} \sim 10^{-2}$ and, hence, that inertial effects are indeed negligible), we write

$$
\frac{\partial}{\partial x}\left(\alpha \sigma_{c}\right)+f_{c}=0 \quad \text { and } \quad \frac{\partial}{\partial x}\left(\beta \sigma_{w}\right)+f_{w}=0 .
$$

In equations (3), $f_{c}$ and $f_{w}$ represent the net sources of momentum in each phase.

Finally, we assume that oxygen transport through the tumour is controlled by diffusion [2], and the quasi-steady equation for the oxygen tension $C(x, t)$ reads

$$
\frac{\partial^{2} C}{\partial x^{2}}=Q_{c}
$$

where $Q_{c}$ represents the rate of oxygen consumption by the tumour cells.

\subsection{Constitutive laws}

Volume source terms

In an arbitrary control volume within an avascular tumour, volume cannot be created: rather it is exchanged between the extracellular and cellular phases. Thus we set $q_{w}=-q_{c}$ and choose $q_{c}$ to be consistent with [29]:

$$
q_{c}=\frac{\alpha(1-\alpha) S_{0} C}{1+S_{1} C}-\frac{S_{2}+S_{3} C}{1+S_{4} C} \alpha .
$$

The first term in (5) represents cell growth due to mitosis, characterised by the parameters $S_{0}$ and $S_{1}$, and the second represents cell death, parameterised by $S_{2}, S_{3}$ and $S_{4}$. We assume throughout the paper that $S_{2}<S_{3} / S_{4}$. We note that the rate of cell growth increases with $C$ and that, with the restriction on $S_{2}$ given above, the rate of cell death increases as $C$ decreases (this is in line with experimental observations, [20]). We do not distinguish different mechanisms of cell death (for example, apoptosis and necrosis); we assume both effects are accounted for in our generic death term.

Stress tensors

We suppose that the stresses in the water and cell phases are given by

$$
\sigma_{w}=-p_{w} \quad \text { and } \quad \sigma_{c}=-p_{c}+2 \mu_{c} \frac{\partial u_{c}}{\partial x} .
$$


In equations $(6), p_{w}$ and $p_{c}$ are the average pressures in the water and cellular phases respectively and $\mu_{c}$ represents the viscosity of the cells. Recall from the introduction that we associate the viscosity of the cellular phase with the degree of differentiation of the tumour cells. Well-differentiated tumours are characterised by significant levels of inter-cellular cohesion (as compared to poorly-differentiated tumours) and thus are have a higher viscosity. In order to close the model equations we specify the following constitutive relationship between the two pressures:

$$
p_{c}=p_{w}+\Sigma_{c},
$$

where $\Sigma_{c}$ describes the pressure in the cell phase due to cell-cell interactions. We suppose that there is a certain volume fraction of cells, $\alpha^{*}$ say, at which the cells are at their natural, close-packing density. At higher volume fractions, the cells' membranes deform and the cells experience stress. This pressure becomes infinite as the cell volume fraction approaches $\alpha=1$. Under such conditions (when $\alpha>\alpha^{*}$ ), the cells will attempt to move in order to reduce their stress. If $\alpha<\alpha^{*}$ and the cells are close enough, the filopodia that they extend may come into contact and stimulate the motion of the cells towards each other, i.e. cell aggregation. This is a short-range phenomenon; if the cells are too far apart $\left(\alpha \leq \alpha_{\text {min }}\right.$ say), they will not experience this attraction. Note that $\alpha_{\min } \leq \alpha^{*}$, with equality holding in the case where there are no short-range forces present between cells. Combining these ideas, we choose the following functional form for $\Sigma_{c}$ :

$$
\Sigma_{c}=\left\{\begin{array}{ccc}
\gamma \frac{\alpha-\alpha^{*}}{(1-\alpha)^{2}} & \text { if } & \alpha>\alpha_{\min } \\
0 & \text { if } & \alpha<\alpha_{\min } .
\end{array}\right.
$$

In (8), we call $\gamma$ the tension constant. It is a measure of the cells' affinity for the natural density, $\alpha=\alpha^{*}$.

Momentum source terms

We suppose that there are momentum sources due to the interfacial pressures and that differential movement of the two phases manifests itself as a Darcy-style drag term. We set

$$
f_{c}=p_{w} \frac{\partial \alpha}{\partial x}+k_{1} \alpha \beta\left(u_{w}-u_{c}\right) \quad \text { and } \quad f_{w}=p_{w} \frac{\partial \beta}{\partial x}-k_{1} \alpha \beta\left(u_{w}-u_{c}\right) .
$$

In (9) $k_{1}$ is the drag coefficient, which is proportional to the viscosity of the extracellular water. We note that additional terms could be included to describe, for example, the change in momentum associated with mass transfer between the two phases [6]. We discuss introducing such terms in Section 6. We have assumed that the drag terms are proportional to the product $\alpha \beta$ (i.e. there is no drag if either species is not present). Other permeabilities may be more physically realistic (for example, we could use the Carman-Kozeny relationship presented in [14]). However, we proceed with our form for the drag because we believe it is the simplest possible form.

Oxygen consumption rate

For consistency with $[3,30]$, we suppose that the oxygen consumption rate is governed by a Michaelis-Menten law $[11]$, and thus we set

$$
Q_{c}=\frac{Q_{0} \alpha C}{1+Q_{1} C}
$$

where $Q_{0}$ and $Q_{1}$ are positive constants.

\subsection{Initial and boundary conditions}

We suppose that the width of the tumour $l(t)$ and the initial volume fraction of the cells are known, so that

$$
l=L_{0} \quad \text { and } \quad \alpha=\alpha_{0} \quad \text { at } \quad t=0 .
$$

We assume symmetry about the tumour centre, so that

$$
\begin{aligned}
u_{c}=u_{w} & =0 \text { at } x=0, \\
\frac{\partial C}{\partial x} & =0 \text { at } x=0 .
\end{aligned}
$$


At the tumour boundary, we assume continuity of stress, and we allow the free passage of water. Neglecting the medium outside the tumour, we therefore have

$$
p_{w}=0, \quad 2 \mu_{c} \frac{\partial u_{c}}{\partial x}=\left\{\begin{array}{ccc}
\gamma \frac{\alpha-\alpha^{*}}{(1-\alpha)^{2}} & \text { if } \quad \alpha>\alpha_{\min }, \quad \text { if } \quad \alpha<\alpha_{\min } .
\end{array} \quad \text { at } \quad x=l\right.
$$

We also assume that the tumour's outer boundary moves at the same speed as the tumour cells on the boundary and so we fix

$$
\frac{\mathrm{d} l}{\mathrm{~d} t}=\left.u_{c}\right|_{x=l}
$$

Finally, we assume that the oxygen tension outside the tumour is constant, and thus we set

$$
C=C_{\text {out }} \quad \text { at } \quad x=l .
$$

In summary, our model consists of the conservation of mass equations (1), with sources given by (5), the no-voids condition (2), the conservation of momentum equations (3), with stresses defined in (6) and sources defined in (9), the constitutive relationship given in (7), the oxygen tension equation (4) with consumption rate given by (10), and the initial and boundary conditions given in (11-16). We note that the model comprises seven equations for the seven unknowns $\alpha, \beta, u_{c}, u_{w}, p_{c}, p_{w}$ and $C$. In the next section we show how the model may be reduced to three equations for $\alpha, u_{c}$ and $C$.

\section{Model simplification}

In this section we reduce the model to a trio of equations for $\alpha, u_{c}$ and $C$. First we add equations (1), to obtain

$$
\frac{\partial}{\partial x}\left(\alpha u_{c}+(1-\alpha) u_{w}\right)=0
$$

which we may integrate to obtain

$$
u_{w}=-\frac{\alpha u_{c}}{1-\alpha},
$$

imposition of (12) indicating that the constant of integration be set to zero. Similarly, adding equations (3), and substituting for the stress in each phase using (6), we find that

$$
\gamma \frac{\partial}{\partial x}\left(\alpha \frac{\alpha-\alpha^{*}}{(1-\alpha)^{2}} H\left(\alpha-\alpha_{m i n}\right)\right)+\frac{\partial p_{w}}{\partial x}=2 \mu_{c} \frac{\partial}{\partial x}\left(\alpha \frac{\partial u_{c}}{\partial x}\right) .
$$

We use the second of equations (3) and (18) to obtain a relationship between $p_{w}$ and $u_{c}$ :

$$
u_{c}=\frac{(1-\alpha)}{k_{1} \alpha} \frac{\partial p_{w}}{\partial x} .
$$

We substitute from (20) into (19) to obtain

$$
\gamma \frac{\partial}{\partial x}\left(\alpha \frac{\alpha-\alpha^{*}}{(1-\alpha)^{2}} H\left(\alpha-\alpha_{m i n}\right)\right)+\frac{k_{1} \alpha u_{c}}{1-\alpha}=2 \mu_{c} \frac{\partial}{\partial x}\left(\alpha \frac{\partial u_{c}}{\partial x}\right) .
$$

Our reduced model may now be stated as comprising the first of equations (1) coupled with equations (4) and (19), with boundary and initial conditions specified by (11-16). 


\subsection{Nondimensionalisation}

We scale the independent and dependent variables in the following manner:

$$
\begin{gathered}
t=\left(1+S_{1} C_{\text {out }}\right) /\left(S_{0} C_{\text {out }}\right) t^{\prime}, \quad x=L_{0} x^{\prime}, \quad l=L_{0} l^{\prime}, \\
\alpha=\alpha^{\prime}, \quad u_{c}=L_{0} S_{0} C_{\text {out }} /\left(1+S_{1} C_{\text {out }}\right) u_{c}{ }^{\prime}, \quad C=C_{\text {out }} C^{\prime} .
\end{gathered}
$$

Omitting the primes, the resulting equations for the dependent variables read,

$$
\begin{gathered}
\frac{\partial \alpha}{\partial t}+\frac{\partial}{\partial x}\left(u_{c} \alpha\right)=\frac{\left(1+s_{1}\right) \alpha(1-\alpha) C}{1+s_{1} C}-\frac{s_{2}+s_{3} C}{1+s_{4} C} \alpha \\
\frac{\partial}{\partial x}\left(\alpha \frac{\alpha-\alpha^{*}}{(1-\alpha)^{2}} H\left(\alpha-\alpha_{\min }\right)\right)+\frac{k \alpha u_{c}}{1-\alpha}=\mu \frac{\partial}{\partial x}\left(\alpha \frac{\partial u_{c}}{\partial x}\right), \\
\frac{\partial^{2} C}{\partial x^{2}}=\frac{Q \alpha C}{1+\hat{Q}_{1} C}
\end{gathered}
$$

with initial and boundary conditions

$$
\begin{aligned}
& l=1, \alpha=\alpha_{0} \text { at } t=0, \\
& u_{c}=0=\frac{\partial C}{\partial x} \text { at } x=0 \text {, } \\
& \mu \frac{\partial u_{c}}{\partial x}=\frac{\alpha-\alpha^{*}}{(1-\alpha)^{2}} H\left(\alpha-\alpha^{*}\right), \quad C=1 \text { at } x=l, \\
& \frac{\mathrm{d} l}{\mathrm{~d} t}=u_{c} \text { at } x=l \text {. }
\end{aligned}
$$

In equations (22)-(27), we have introduced

$$
\begin{array}{ll}
s_{1}=S_{1} C_{\text {out }}, \quad s_{2}=S_{2} \frac{1+S_{1} C_{\text {out }}}{S_{0} C_{\text {out }}}, \quad s_{3}=S_{3} \frac{1+S_{1} C_{\text {out }}}{S_{0}}, \quad s_{4}=S_{4} C_{\text {out }}, \\
k=\frac{k_{1} L_{0}^{2} S_{0} C_{\text {out }}}{\gamma\left(1+S_{1} C_{\text {out }}\right)}, \quad \mu=\frac{2 \mu_{c} S_{0} C_{\text {out }}}{\gamma\left(1+S_{1} C_{\text {out }}\right)}, \quad Q=Q_{0} L_{0}^{2}, \quad \hat{Q}_{1}=Q_{1} C_{\text {out }} .
\end{array}
$$

In this study, we shall focus our attention on the effects of varying the viscosity parameter $\mu$ and the drag constant $k$.

\subsection{Change of variables}

Before carrying out the numerical solutions, it is convenient to map the domain of interest onto a fixed domain. We change variables from $(x, t)$ to $(\xi, \tau)$, where $\xi=x / l(t)$ and $\tau=t$. The transformed problem reads

$$
\begin{gathered}
\frac{\partial \alpha}{\partial \tau}-\frac{\xi}{l} \frac{\mathrm{d} l}{\mathrm{~d} \tau} \frac{\partial \alpha}{\partial \xi}+\frac{1}{l} \frac{\partial}{\partial \xi}\left(u_{c} \alpha\right)=\frac{\left(1+s_{1}\right) \alpha(1-\alpha) C}{1+s_{1} C}-\frac{s_{2}+s_{3} C}{1+s_{4} C} \alpha \\
l \frac{\partial}{\partial \xi}\left(\alpha \frac{\alpha-\alpha^{*}}{(1-\alpha)^{2}} H\left(\alpha-\alpha_{\min }\right)\right)+\frac{k l^{2} \alpha u_{c}}{1-\alpha}=\mu \frac{\partial}{\partial \xi}\left(\alpha \frac{\partial u_{c}}{\partial \xi}\right) \\
\frac{\partial^{2} C}{\partial \xi^{2}}=\frac{Q l^{2} \alpha C}{1+\hat{Q}_{1} C}
\end{gathered}
$$

with initial and boundary conditions 


$$
\begin{array}{r}
l=1, \alpha=\alpha_{0} \text { at } \tau=0, \\
u_{c}=0=\frac{\partial C}{\partial \xi} \text { at } \xi=0, \\
\mu \frac{\partial u_{c}}{\partial \xi}=l \frac{\alpha-\alpha^{*}}{(1-\alpha)^{2}} H\left(\alpha-\alpha_{\min }\right), \quad C=1 \text { at } \xi=1, \\
\frac{\mathrm{d} l}{\mathrm{~d} \tau}=u_{c} \text { at } \xi=1 .
\end{array}
$$

Before presenting numerical simulations of the model equations, there are several points to note. In the case where viscous effects are negligible, $\mu \rightarrow 0$ and the stress equation (30) reveals that

$$
u_{c}=-\frac{1-\alpha}{k l \alpha} \frac{\partial}{\partial \xi}\left(\frac{\alpha\left(\alpha-\alpha^{*}\right)}{(1-\alpha)^{2}} H\left(\alpha-\alpha_{\min }\right)\right) .
$$

Substituting with (36) in (29) we deduce

$$
\frac{\partial \alpha}{\partial \tau}=\frac{1}{k l^{2}} \frac{\partial}{\partial \xi}\left[(1-\alpha) \frac{\partial}{\partial \xi}\left(\frac{\alpha\left(\alpha-\alpha^{*}\right)}{(1-\alpha)^{2}} H\left(\alpha-\alpha_{m i n}\right)\right)\right]+\frac{\xi}{l} \frac{\mathrm{d} l}{\mathrm{~d} \tau} \frac{\partial \alpha}{\partial \xi}+\left(\frac{\left(1+s_{1}\right) \alpha(1-\alpha) C}{1+s_{1} C}-\frac{s_{2}+s_{3} C}{1+s_{4} C} \alpha\right)
$$

with

$$
\begin{aligned}
& \alpha=\alpha_{0}, \quad l=1 \text { at } \tau=0, \\
& \frac{\partial \alpha}{\partial \xi}=0 \text { at } \xi=0, \\
& \alpha=\alpha^{*} \text { at } \xi=1, \\
& \frac{\mathrm{d} l}{\mathrm{~d} \tau}=\frac{1}{k l\left(1-\alpha^{*}\right)} \frac{\partial \alpha}{\partial \xi} \text { at } \xi=1 .
\end{aligned}
$$

Thus, in the limit as $\mu \rightarrow 0$ the problem reduces to a nonlinear diffusion equation for the tumour cell volume fraction, $\alpha$. Such equations have been proposed by other authors to describe tumour growth (see, for example, $[15,25,26])$. Whereas Sherratt, Gatenby and others used nonlinear diffusion equations as the starting point for their modelling and analysis, we have presented a systematic argument, based on cellular behaviour, which justifies their description of cellular motion as a diffusion process. Note that, while equation (30) is formally singular in the limit $\mu \rightarrow 0$, no boundary layer is formed because we are fortunate enough to still be able to satisfy all the boundary conditions in this limit.

By relaxing the assumption that viscous effects are negligible, so that $\mu>0$, we can generate a differential equation for $A(\tau)=\alpha(l(\tau), \tau)$ :

$$
\frac{\partial A}{\partial \tau}+\frac{A}{\mu}\left(\frac{\left(A-\alpha^{*}\right) H\left(A-\alpha_{m i n}\right)}{(1-A)^{2}}\right)=A\left(1-s_{5}-A\right),
$$

where $s_{5}=\left(s_{2}+s_{3}\right) /\left(1+s_{4}\right)$. Note that $s_{5}$ denotes the ratio of the tumour cell death rate to the tumour cell birth rate that occurs when the oxygen tension is at the externally supplied level, (i.e. when $C=C_{\text {out }}$ ). In Figure 1 we sketch $A(\tau)$ as $\tau$ varies for different choices of $\mu$ and fixed values of $s_{5}$ and $A(0)$. From these results we note that as the cells become more viscous (i.e. as $\mu$ increases) the time taken for $A$ to reach its steady state increases. Also, we see that, for all parameter values considered, $A$ has attained its steady state by time $\tau=1$. Now, the steady state, $A_{s}=A(\tau \rightarrow \infty)$, satisfies

$$
A_{s}=0, \quad \text { or } \quad \frac{1}{\mu} \frac{\left(A_{s}-\alpha^{*}\right)}{\left(1-A_{s}\right)^{2}} H\left(A_{s}-\alpha_{\min }\right)=1-s_{5}-A_{s} .
$$

If $1-s_{5}<\alpha_{\min }$, then $A_{s}=1-s_{5}$ whereas if $1-s_{5}>\alpha_{\text {min }}$, then $A_{s}>\alpha_{\min }$ solves

$$
0=\mu\left(1-A_{s}\right)^{3}-\mu s_{5}\left(1-A_{s}\right)^{2}+\left(1-A_{s}\right)+\alpha^{*} .
$$


Figures 1 and 2 near here

In Figure 2 we plot the physically relevant root of the above cubic as $\mu$ varies, for fixed values of $s_{5}$. We note that if $1-s_{5}>\alpha^{*}$ then $A_{s}>\alpha^{*}$ so that the cells at the tumour's edge are in a stressed state, and we expect the tumour to grow. From (43) we deduce that $A_{s} \rightarrow \alpha^{*}$ as $\mu \rightarrow 0$, and $A_{s} \rightarrow 1-s_{5}$ as $\mu \rightarrow \infty$. Further, when $s_{5}=1 / 11$ and $\mu=1$, we find that $A_{s}=0.804$, and, so, $A_{s}-\alpha^{*} \ll 1$. In the limit $s_{5} \sim 1-\alpha^{*}$, we consider the expansion $A_{s}=\alpha^{*}+\epsilon \bar{A}$, where $\epsilon=1-\alpha^{*}-s_{5}$. Substituting this form into (43), we find that

$$
\frac{\epsilon \bar{A}}{\mu\left(1-\alpha^{*}-\epsilon \bar{A}\right)^{2}}=\epsilon(1-\bar{A}) \quad \Rightarrow \quad \bar{A}=\frac{\mu\left(1-\alpha^{*}\right)^{2}}{1+\mu\left(1-\alpha^{*}\right)^{2}}+O(\epsilon)
$$

We can use the relationship for $A_{s}$ in (34) to determine $\partial u_{c} / \partial \xi$ at $\xi=1$ :

$$
\frac{\partial u_{c}}{\partial \xi}(\xi=1, \tau) \sim \frac{\epsilon l}{1+\mu\left(1-\alpha^{*}\right)^{2}}+O\left(\epsilon^{2}\right) .
$$

Thus, since we know, from the simulations presented in Figure 1, that $A$ approaches its steady state by $\tau \sim 1$, when studying the longtime behaviour of the system it is appropriate to scale $u_{c}=\epsilon u$ and $\tau=T / \epsilon$ : this scaling ensures a nontrivial balance in (35).

In the case where we neglect $\operatorname{drag}($ i.e. $k \rightarrow 0)$, which corresponds to the situation where the cells are not affected by the flow of extracellular water between them, spatial variations in the water pressure become negligible and the model reduces to a single equation for $\alpha$ : we integrate (30) twice to find that

$$
u_{c}=\frac{l}{\mu} \int_{0}^{\xi} \frac{\alpha-\alpha^{*}}{(1-\alpha)^{2}} H\left(\alpha-\alpha_{m i n}\right) d s .
$$

Substituting (46) into (29) we find that

$$
\begin{aligned}
\frac{\partial \alpha}{\partial \tau}+\frac{\alpha}{\mu} \frac{\alpha-\alpha^{*}}{(1-\alpha)^{2}} H\left(\alpha-\alpha_{\text {min }}\right)+ & \frac{1}{\mu} \frac{\partial \alpha}{\partial \xi} \int_{0}^{\xi} \frac{\alpha-\alpha^{*}}{(1-\alpha)^{2}} H\left(\alpha-\alpha_{m i n}\right) d s-\frac{\xi}{l} \frac{\mathrm{d} l}{\mathrm{~d} \tau} \frac{\partial \alpha}{\partial \xi} \\
& =\left(\frac{\left(1+s_{1}\right) \alpha(1-\alpha) C}{1+s_{1} C}-\frac{s_{2}+s_{3} C}{1+s_{4} C} \alpha\right) .
\end{aligned}
$$

The initial and boundary conditions read

$$
\begin{array}{rlrl}
\alpha=\alpha_{0}, \quad l=1 & \text { at } & \tau=0, \\
\alpha=A & \text { at } & \xi=1, \\
\frac{\mathrm{d} l}{\mathrm{~d} \tau}=\frac{l}{\mu} \int_{0}^{1} \frac{\alpha-\alpha^{*}}{(1-\alpha)^{2}} H\left(\alpha-\alpha_{\min }\right) d s & \text { at } & \xi & =1 .
\end{array}
$$

Thus, in the limit $k \rightarrow 0$, the problem reduces to a non-local, integro-differential equation for the tumour cell volume fraction, $\alpha$. To our knowledge, this is the first time such a model has been proposed to describe tumour growth.

In the case where both viscous effects and the drag are negligible $($ i.e. $\mu \rightarrow 0, k \rightarrow 0$ ), the momentum balance equation (30) predicts that the tumour cell volume fraction is constant, and equal to $\alpha^{*}$, from the boundary conditions. Equation (29) then defines the speed with which the tumour cells move:

$$
\frac{\partial u_{c}}{\partial \xi}=l\left(\frac{\left(1+s_{1}\right)\left(1-\alpha^{*}\right) C}{1+s_{1} C}-\frac{s_{2}+s_{3} C}{1+s_{4} C}\right) .
$$

In (51), the evolution of $l$ is governed by (35) so that

$$
\frac{\mathrm{d} l}{\mathrm{~d} \tau}=\left.u_{c}\right|_{\xi=1}=l \int_{0}^{1}\left\{\frac{\left(1+s_{1}\right)\left(1-\alpha^{*}\right) C}{1+s_{1} C}-\frac{s_{2}+s_{3} C}{1+s_{4} C}\right\} d \xi .
$$

Thus, in the limit as $k, \mu \rightarrow 0$, our model describes a situation in which $\alpha$ is constant and the tumour's growth rate depends on the oxygen distribution. This reduced model is similar in form to models presented by Greenspan [16] and Landman and Please [22]. 
Finally, we note that in the limit in which the (dimensional) tension constant $\gamma$ is negligible, (i.e. $\mu, k \rightarrow$ $\infty)$, condition (34) reduces to $u_{c \xi}=0$, and the solution to (30), (33), (34) and (35) is $u_{c}=0, l=1$. In this case, the tumour does not grow and (29) reduces to an ordinary differential equation that describes how mass is exchanged between the cellular and extracellular phases, until the volume fraction is such that the cells experience repulsive forces and the tumour can increase in size.

\section{Numerical simulations}

In this section we present numerical simulations of equations (29)-(35), scaled so that we use $u$ and $T$ rather than $u_{c}$ and $\tau$ (as described in the last section), in several parameter regimes. Our aims are to determine the general behaviour of the model equations and to see how varying two key model parameters, namely the viscosity constant and the drag constant, affects the tumour's growth.

In the absence of a complete set of parameter values, we focus on the qualitative nature of the model equations and pick the following values of the parameters:

$$
\begin{gathered}
Q=0.5, \quad \hat{Q}_{1}=0, \quad s_{1}=s_{4}=10, \quad s_{2}=s_{3}=0.5, \\
k=1=\mu=1, \quad \alpha_{0}=\alpha_{\text {min }}=\alpha^{*}=0.8 .
\end{gathered}
$$

We have taken $\alpha^{*}=\alpha_{\min }$ so that the effects of cellular attraction are not included in this simulation. We show how $\alpha, u$ and $l$ evolve over time in Figures 3a, b and c respectively. We find that, initially, the tumour cell volume fraction $\alpha$ increases towards the centre of the tumour (results not shown, but see Section 7 for an explanation): the tumour can relieve the stress at its outer boundary by expanding outwards and, thus, reducing the volume fraction there. Following this early phase, the tumour becomes sufficiently large that cells at the centre start to die, so that a central necrotic core forms and $\alpha$ there decreases. The system then settles to a steady, travelling-wave solution, in which cells close to the edge of tumour move outwards (to relieve their stress), whilst cells nearer to the tumour centre move inwards. Figure 3c confirms that the system settles to a steady, travelling-wave profile: after an initial period of growth, the speed with which the leading edge of the tumour advances is approximately constant.

Figure 3 about here

In Figure 4 we show how the size of the tumour changes over time, for different choices of the initial volume fraction $\alpha(x, 0)=\alpha_{0}$. For $\alpha_{0}<\alpha_{\min }$ ( $=\alpha^{*}$ in this case), there is a "waiting time" during which the tumour does not change in size but the tumour cell volume fraction increases to $\alpha^{*}$ at the tumour's edge.

Figure 4 about here

The results presented in Figure 5 show how the tumour's growth alters when the drag parameter $k$ is changed: as the drag coefficient increases the tumour's growth rate reduces. The drag parameter represents the ease with which extracellular water may pass between the tumour cells. As this parameter is increased, the extracellular water is less able to flow between the cells and hence the mass available for tumour cell proliferation diminishes.

Figure 5 about here

The impact that changing the viscosity parameter $\mu$ has on the tumour's growth is depicted in Figure 6 . We observe that as $\mu$ increases the tumour's growth rate reduces. The viscosity parameter represents the amount of inter-cellular cohesion, which is high for well-differentiated tumours, and low for poorlydifferentiated tumours. Increasing this parameter corresponds to stronger inter-cellular bonds. 
Figure 6 about here

In Figure 7 we investigate the effects of altering $\mu$ and $k$ simultaneously, in a way that corresponds to changing the tension constant $\gamma$ (see definition of dimensionless parameter groups $k$ and $\mu$ following equation (28)). Decreasing $k$ and $\mu$ together (i.e. increasing $\gamma$ ), increases the speed of tumour growth. The tension constant corresponds to the affinity that the cells have for their natural volume fraction. As this parameter increases, the cells move faster in order to try and regain the natural volume fraction. We discuss the physical interpretations of these observations further in section 6 .

Figure 7 about here

Finally we relax the assumption that $\alpha_{\min }=\alpha^{*}$ and consider the impact of fixing $\alpha_{\min }<\alpha^{*}$. Physically, this means that the cells sense each other using their filopodia, and are attracted towards each other. When $\alpha_{\text {min }}=0.6$, the system settles to a steady state. The resulting steady state profiles for $\alpha$ and $u$, together with the evolution of the tumour boundary $l=l(T)$, are presented in Figure 8. In this steady state, the motion of the cells is everywhere inward, and the cellular volume fraction is significant everywhere. We do not observe the formation of an area of necrosis.

Figure 8 about here

\section{Analysis}

In this section we present some analytical results which help to explain some of the behaviour observed in the last section. In particular, we derive an expression for the "waiting time" depicted in Figure 4, necessary conditions for the formation of a steady state solution, and we investigate oxygen-independent growth (corresponding to the initial growth of the tumour) for different limits of the cellular viscosity $\mu$.

\subsection{Transient analysis for tumours seeded below $\alpha_{\text {min }}$}

When $\alpha(\xi, 0)=\alpha_{0}<\alpha_{\text {min }}$, the solution to (30) is $u_{c}=0$. Equation (29) then reduces to a time-dependent ordinary differential equation which describes the evolution of $\alpha$ at each position $x$. The tumour will remain of size $l=1$ until $\tau=\tau_{\text {wait }}$, at which time $\alpha(1, \tau)=\alpha_{\text {min }}$. We may determine this "waiting time" by solving the differential equation for $\alpha(1, \tau)$. With $u_{c}=0$, equation (42) reduces to

$$
\frac{\partial A}{\partial \tau}=A\left(1-A-s_{5}\right), \quad A(0)=\alpha_{0}
$$

with solution

$$
A=\frac{\left(1-s_{5}\right) \alpha_{0} e^{\left(1-s_{5}\right) \tau}}{1-s_{5}-\alpha_{0}-\alpha_{0} e^{\left(1-s_{5}\right) \tau}} .
$$

Thus, the waiting time, $\tau_{\text {wait }}$, is given by

$$
\tau_{\text {wait }}=\frac{1}{1-s_{5}} \log \left(\frac{\alpha_{\min }\left(1-s_{5}-\alpha_{0}\right)}{\alpha_{0}\left(1-s_{5}-\alpha_{\min }\right)}\right) .
$$

Remembering that $T=\left(1-s_{5}-\alpha^{*}\right) \tau$, we show how the waiting time $T_{w a i t}$ varies with $\alpha_{0}$ in Figure 9 . There is good agreement between the analytical values and the numerical estimates of Figure 4. 
Figure 9 about here

\subsection{Necessary conditions for realising an equilibrium solution}

The numerical results presented in Figure 8 suggest that, in certain parameter regimes, the system ultimately evolves to a steady state. We suppose that such a state is possible, and seek necessary conditions for realising equilibrium solutions. If a steady state occurs then $u_{c}=0$ at $\xi=1$. Integrating equation (34) and noting that $u_{c}=0$ at $\xi=0,1$, we deduce that a necessary condition for obtaining a steady state solution is:

$$
\int_{0}^{1}\left\{\frac{\alpha(1-\alpha)\left(1+s_{1}\right) C}{1+s_{1} C}-\frac{s_{2}+s_{3} C}{1+s_{4} C} \alpha\right\} d \xi=0 .
$$

Integrating equation (30) twice with respect to $\xi$ we find that a second necessary condition is:

$$
\int_{0}^{1} \frac{\alpha\left(\alpha-\alpha^{*}\right) H\left(\alpha-\alpha_{\min }\right)}{(1-\alpha)^{2}} d x=\int_{0}^{1} \frac{k}{\alpha} \int_{\xi}^{1} \frac{\alpha u_{c}}{1-\alpha} d y d x .
$$

Since $u_{c} \leq 0$ and $0 \leq \alpha \leq 1$, we note that the integral on the right-hand-side (RHS) of (56) is non-positive and, for non-trivial solutions, it is negative. Since the RHS of (56) is non-zero, we deduce that $\alpha>\alpha_{\text {min }}$ for a finite range of points within the tumour volume. Since, for a nontrivial solution, the integrals in (56) are both negative, we deduce further that $\alpha^{*}>\alpha_{\min }$ is a necessary condition for obtaining a steady state solution. We note that the numerical simulations presented in section 4 are consistent with these necessary conditions. The physical interpretation is that, in order for our tumour to reach a steady state, we must have a region within the tumour where the cells are sparsely populated and short-range attractive forces are acting in order to balance the repulsive forces experienced in denser packed regions of the tumour.

\subsection{Analysis for initial stages of tumour growth}

In this section we focus on equations (22)-(28) during the initial stages of avascular tumour growth, when all cells are well-oxygenated and, hence, proliferate at the maximal, oxygen-independent rate. The asymptotic limit of interest has $Q=\hat{Q}_{1}=0$ (and, hence, $C \equiv 1$ ) and $s_{2}=s_{3}=0$ (and, hence, no cell death). In what follows, to simplify the analysis, we shall also assume that $0<1-\alpha^{*} \ll 1$. Physically, this corresponds to the case where, at the natural packing density, the tumour cells fill almost all of the available space within the tumour.

We scale $\alpha, u_{c}$ and $t$ using similar scalings to those proposed at the end of section 3. Specifically, we write

$$
1-\alpha=\epsilon \theta, \quad u_{c}=\epsilon u, \quad t=T / \epsilon, \quad \text { where } \quad 0<\epsilon=1-\alpha^{*} \ll 1 .
$$

Under these rescalings, equations (22) and (23) become

$$
\begin{gathered}
-\epsilon \frac{\partial \theta}{\partial T}+\frac{\partial}{\partial x}((1-\epsilon \theta) u)=(1-\epsilon \theta) \theta \\
\epsilon \mu \frac{\partial}{\partial x}\left((1-\epsilon \theta) \frac{\partial u}{\partial x}\right)-\frac{\partial}{\partial x}\left((1-\epsilon \theta) \frac{(1-\theta)}{\epsilon \theta^{2}}\right)=k \frac{1-\epsilon \theta}{\theta} u
\end{gathered}
$$

with boundary conditions

$$
\begin{aligned}
u=0 \text { at } x & =0, \\
\mu \frac{\partial u}{\partial x}=\frac{(1-\theta)}{\epsilon^{2} \theta^{2}} \text { at } x & =l, \\
\frac{\mathrm{d} l}{\mathrm{~d} T}=u \text { at } x & =l .
\end{aligned}
$$

If we introduce $\nu=\mu \epsilon^{2}, \kappa=k \epsilon$ then equations (57) and (58) read (at leading-order in $\epsilon$ )

$$
\frac{\partial u}{\partial x}=\theta, \quad \nu \frac{\partial^{2} u}{\partial x^{2}}+\frac{(2-\theta)}{\theta^{3}} \frac{\partial \theta}{\partial x}=\kappa \frac{u}{\theta},
$$


and may be combined to yield a single equation for $\theta$ :

$$
\frac{\partial}{\partial x}\left(\left(\nu \theta+\frac{2}{\theta^{2}}-\frac{1}{\theta}\right) \frac{\partial \theta}{\partial x}\right)=\kappa \theta
$$

The boundary conditions now read

$$
\begin{aligned}
& \frac{\partial \theta}{\partial x}=0 \text { at } x=0, \\
& \nu \theta=\frac{(1-\theta)}{\theta^{2}} \text { at } x=l, \\
& \frac{\mathrm{d} l}{\mathrm{~d} T}=\frac{1}{\kappa}\left(\nu \theta+\frac{2}{\theta^{2}}-\frac{1}{\theta}\right) \frac{\partial \theta}{\partial x} \text { at } x=l .
\end{aligned}
$$

We now investigate the behaviour of equations (62)-(65) for three limiting cases: (a) $0<\nu \ll 1$, (b) $\nu \sim O(1)$ and (c) $\nu \gg 1$. Case (a) corresponds to a situation in which the viscous effects are unimportant, and the tumour development is governed by drag and tension effects, case (b) corresponds to a situation in which viscous, tension and drag effects are all important, and case (c) corresponds to a situation in which viscous effects dominate.

(a) Small $\nu$ limit. When $0<\nu \ll 1$, viscous effects can be neglected and the leading-order problem for $\theta$ may be integrated once by introducing $\partial \theta / \partial x=\varphi(\theta)^{1 / 2}$ and imposing (63). We find that

$$
\varphi=\frac{2 \kappa \theta^{4}}{(2-\theta)^{2}}\left(2 \log \left(\theta / \theta^{*}\right)+\left(\theta^{*}-\theta\right)\right),
$$

where $\theta^{*}(T)=\theta(0, T)$. Referring to (64), we remark that, since $0<\nu \ll 1, \theta=1$ at $x=l$ and, so, (65) implies that

$$
\kappa\left(\frac{\mathrm{d} l}{\mathrm{~d} T}\right)^{2}=-4 \log \theta^{*}-2\left(1-\theta^{*}\right)
$$

Using the solution for $\varphi$ given above, we find that the (implicit) solution for $\theta$ reads

$$
\sqrt{2 \kappa} x=\int_{\theta^{*}}^{\theta} \frac{(2-s) d s}{s^{2} \sqrt{2 \log \left(s / \theta^{*}\right)-\left(s-\theta^{*}\right)}} .
$$

It remains to determine the size of the tumour $l$ and $\theta^{*}$. There are two equations for these two unknowns, namely (67) and

$$
\sqrt{2 \kappa} l=\int_{\theta^{*}}^{1} \frac{(2-s) d s}{s^{2} \sqrt{2 \log \left(s / \theta^{*}\right)-\left(s-\theta^{*}\right)}} .
$$

Combining these equations yields the following equation for $\theta^{*}$ :

$$
\frac{\partial \theta^{*}}{\partial T}=2\left(-2 \log \theta^{*}-\left(1-\theta^{*}\right)\right) \theta^{*^{2}}+\left(-2 \log \theta^{*}-\left(1-\theta^{*}\right)\right)^{1 / 2} \theta^{* 2} \int_{1}^{1 / \theta^{*}} \Lambda\left(r, \theta^{*}\right) d r
$$

where

$$
\Lambda\left(r, \theta^{*}\right)=\frac{16 \log r-2(-5+5 r+2 r \log r) \theta^{*}+3(r-1) r \theta^{* 2}}{2 r^{2} \theta^{*}\left(-2 \log r+(r-1) \theta^{*}\right) \sqrt{2 \log r+\theta^{*}(1-r)}} d r .
$$

In practice, it is easier to solve (66) numerically, treating $l$ as a parameter and identifying the value of $\theta^{*}$ that is consistent with the boundary conditions and this choice of $l$. The solutions for various values of $l$ are presented in Figure 10. We see that, as the tumour grows, $\theta$ decreases internally and has a minimum at the centre of the tumour. Thus, as the tumour grows, the tumour cell volume fraction $(\alpha=(1-\theta) / \epsilon)$ increases internally, and has a maximum at the centre. We can understand this behaviour by considering the 
following argument. Cells throughout the tumour mass proliferate. In the vicinity of the boundary, there is less resistance to motion than in the centre and so the cellular volume fraction is lower near the boundary than in the centre due to cell motion. The tumour mass expands because the cellular volume fraction exceeds the natural packing density throughout, and hence the tumour grows in order to relieve this stress.

When determining how $l$ increases over time, there are two options: either we solve equation (70) to obtain $\theta^{*}$, or we use the value of $\theta^{*}=\theta^{*}(l)$ identified from the numerical solution described above. In both cases, we then use (67) to determine $l$. In practice, we write

$$
\int_{1}^{l} \frac{d s}{\left(-2 \log \left(\theta^{*}(s)\right)-\left(1-\theta^{*}(s)\right)\right)^{1 / 2}}=\sqrt{\frac{2}{\kappa}} \tau,
$$

and use the second approach. The resulting growth curves for different choices of $\kappa$ are presented in Figure 11. They show that increasing $\kappa$ retards tumour growth. Since $\kappa=\epsilon k \propto k_{1} / \gamma$, this corresponds to either increasing the drag coefficient $k_{1}$ or decreasing the tension constant $\gamma$. Thus the qualitative behaviour of our reduced, oxygen-rich model is in good agreement with the numerical results of the full model presented in Figures 5 ( $k$ varies) and 7 ( $\gamma$ varies).

Figures 10 and 11 here

(b) $\nu \sim O(1)$. When $\nu \sim O(1)$, viscous, drag and tension effects are all important. As above, we substitute $\frac{\partial \theta}{\partial x}=\varphi(\theta)^{1 / 2}$ into $(62)$, and find that

$$
\varphi=\frac{2 \kappa \theta^{4}}{\left(2-\theta+\nu \theta^{3}\right)^{2}}\left(2 \log \left(\theta / \theta^{*}\right)-\left(\theta-\theta^{*}\right)+\frac{\nu}{3}\left(\theta^{3}-\theta^{* 3}\right)\right) .
$$

Using the boundary conditions at $x=l$, we find that the condition for $l_{T}$ reads

$$
\frac{\kappa}{2}\left(\frac{\mathrm{d} l}{\mathrm{~d} T}\right)^{2}=2 \log \left(\theta_{l} / \theta^{*}\right)-\left(\theta_{l}-\theta^{*}\right)+\frac{\nu}{3}\left(\theta_{l}^{3}-\theta^{* 3}\right),
$$

where $\theta_{l}$ is the positive root of (64) which decreases monotonically with $\nu$.

In order to ascertain the effects of including viscosity, we compare $\theta$ for tumours of various size in Figure 12 , for $\nu=0$ and $\nu=1$. We fix $\kappa=1$ throughout these calculations. We see that, in each case, the viscosity lowers the value of $\theta$ throughout the tumour, but that, for large $l$, the difference between the viscous and non-viscous cases is negligible except close to the advancing front. We show how the tumour size varies with time in Figure 13. We see that the viscous term retards the tumour's growth, so that, again, the reduced, oxygen-rich model exhibits the same qualitative behaviour as the full model (see Figure 6).

Figures 12 and 13 near here

(c) Large $\nu$ limit. When $\nu \gg 1$, viscous effects dominate drag and tension effects. We set $\delta=1 / \nu \ll 1$, and then the problem reads

$$
\begin{aligned}
& \frac{\partial}{\partial x}\left(\left(\theta+\frac{\delta(2-\theta)}{\theta^{2}}\right) \frac{\partial \theta}{\partial x}\right)=\delta \kappa \theta, \\
& \frac{\partial \theta}{\partial x}=0 \text { at } x=0, \\
& \theta^{3}=\delta(1-\theta) \text { at } x=l, \\
& \delta \frac{\mathrm{d} l}{\mathrm{~d} T}=\frac{1}{\kappa}\left(\theta+\frac{\delta(2-\theta)}{\theta^{2}}\right) \frac{\partial \theta}{\partial x} \text { at } x=l .
\end{aligned}
$$


In this limit, regardless of the size of $\kappa$, the leading-order solution when we take $\delta \rightarrow 0$ is

$$
\theta=0, \quad l=1 .
$$

In the leading-order solution, the tumour mass consists entirely of cells, which cannot proliferate since there is no extracellular water present. The tumour therefore stays at a constant width.

We are forced to rescale $\theta$ so that $\theta \neq 0$ at $x=1$. Using (77), we deduce that the appropriate scaling is $\theta=\delta^{1 / 3} \psi$, and the behaviour of the resulting model depends on the asymptotic size of $\kappa$ relative to $\delta$.

If $\kappa \sim O\left(\delta^{-1 / 3}\right)$, then the drag term is negligible, and the solution to the problem reads

$$
\theta=\nu^{-1 / 3}, \quad l=1 .
$$

If $\kappa \sim O\left(\delta^{-2 / 3}\right)$, we set $K=\delta^{2 / 3} \kappa$, and rescale time in order to retain the time-dependence in equation (78) (specifically, we fix $T=\delta^{-1 / 3} T^{\prime}$ ). As for the cases of small and $O(1)$ viscosity, we are able to integrate the resulting problem. If we set $\varphi^{1 / 2}=\frac{\partial \psi}{\partial x}$, then the equation for $\varphi$ reads

$$
\frac{\partial \varphi}{\partial \psi}-\frac{2\left(4-\psi^{3}\right)}{\psi\left(2+\psi^{3}\right)} \varphi=\frac{2 K \psi^{3}}{2+\psi^{3}},
$$

which has solution

$$
\varphi=\frac{2 K \psi^{4}}{\left(2+\psi^{3}\right)^{2}}\left(2 \log \left(\psi / \psi^{*}\right)+\frac{1}{3}\left(\psi^{3}-\psi^{* 3}\right)\right)
$$

The tumour's growth rate satisfies

$$
\frac{K}{2}\left(\frac{\mathrm{d} l}{\mathrm{~d} T^{\prime}}\right)^{2}=-2 \log \psi^{*}+\frac{1}{3}\left(1-\psi^{* 3}\right) .
$$

We comment that time has been rescaled to a long timescale $T^{\prime}=\delta^{1 / 3} T$. This indicates that the tumour's growth rate slows as $\mu \rightarrow \infty$, a result which is consistent with the numerical results presented in Figure 6 .

As a final remark, we note that, as the tumour gets larger, the effects of oxygen depletion become more important and the validity of our assumption that the tumour is nutrient-rich becomes less valid.

\section{Discussion}

In this paper we have presented a new mathematical model that describes the growth of an avascular tumour. The model is based on a multiphase flow, and incorporates the effects of (i) cell proliferation and death changes that depend on the local oxygen tension, (ii) motion of the cells due to short range attraction (if the cells are sparsely populated) and repulsion (if the cells are overcrowded), (iii) the viscous nature of the cells, (iv) drag forces caused by the motion of extracellular water through the gaps between the cells, and (v) changes in oxygen tension due to cellular consumption. Formulating the model in this way allows us to couple together mechanical and growth phenomena. For example, our framework could easily be used to study the effect of stress on cellular proliferation and death, as demonstrated experimentally in $[9,19]$ and studied in [7].

We were able to solve explicitly for the volume fraction of tumour cells on the tumour boundary and to show that the tumour cell death rate was crucial in determining the behaviour of the advancing front. In particular, if the death rate is less than a threshold value, then cells at the front are in a stressed state and we predict that the tumour will expand in order to relieve this stress. We showed that a number of models for tumour growth that exist in the literature may be recovered as special, limiting cases of our model. For example, when viscous effects are neglected, the resulting model reduces to a nonlinear diffusion equation for the cellular volume fraction (akin to the models presented by Sherratt [25,26] and Gatenby [15]). Thus, our model provides a justification for using nonlinear diffusion equations to describe tumour growth, and provides an interpretation of the diffusivity in such models in terms of cell-cell interactions. Further, when both viscosity and interphase drag were neglected, the model admitted solutions in which the cellular volume fraction was constant and the tumour's growth rate was regulated by the oxygen tension along. This reduced model is in line with models developed by Byrne and Chaplain [5], Greenspan [16] and Landman and Please $[22]$. 
In section 4 we presented numerical simulations of the model equations. We saw that, in the case where the short-range, attractive force was inactive, the tumour developed a necrotic core, and the live cells were confined to a thin region near the advancing front. Cells close to this front propagated outwards, while cells closer to the centre migrated inwards. The system settled to a travelling wave with near constant speed of propagation. The distribution of live and dead cells within the tumour mass mirrors the actual spatial structure observed in a multicell spheroid in vitro (see [24]), and also has the same features as the solutions presented in [28]. Tumours seeded below the natural packing density enjoyed a period of quiescence during which the overall size of the tumour remained fixed and the cell density increased to the natural packing density. Thereafter the tumour expanded, as normal. We were able to predict this waiting time and show how it depended on system parameters such as the initial cell density and the natural packing density. Whilst the existence of waiting times in solid tumour growth remains to be investigated experimentally, we note that this result may have important implications in tissue engineering [8]. Here pieces of tissue, such as cartilage, that are needed to replace damaged or degraded tissue, are cultured in vitro by seeding the relevant cells on a porous scaffold which is bathed in fluid and vital nutrients. We anticipate that in such circumstances there may be an initial quiescent period, or waiting time, during which the seeded cells increase in number but the colony size does not increase in size.

By performing a series of numerical simulations (with the short-range, attractive force switched off), we investigated the impact that changing the model parameters had on the tumour's growth. We found that increasing the drag parameter reduces the speed of tumour growth, increasing the viscosity parameter reduces the tumour growth, while increasing the tension constant increases the speed of tumour growth. Our prediction that increasing the drag parameter reduces the speed of tumour growth is consistent with the interpretation that the drag represents the ease with which extracellular water may pass between the tumour cells, and correlates with the viscosity of the extracellular water (which, in this paper, comprises of water and necrotic debris). Increasing the drag increases the resistance to the motion of extracellular water, and, in turn, this slows local growth of tumour cells due to lack of available building blocks. Our prediction that increasing the cellular viscosity decreases the speed of tumour growth is consistent with the idea that cellular viscosity correlates with the degree of differentiation (and, hence, invasiveness) of the tumour cells, i.e. we predict that tumours containing well-differentiated cells grow more slowly than their less differentiated counterparts. Our prediction that the speed of tumour growth increases with increasing the tension coefficient is consistent with the idea that the tension coefficient corresponds to the affinity of the cells to their natural volume fraction. Increasing the tension coefficient increases this affinity and increases the speed with which the cells move in order to try and regain the natural volume fraction. This results in an increase in the speed of expansion of the tumour when the cells proliferate. We suggest that experiments be carried out to test these hypotheses. For example, to test whether increasing the extracellular drag decreases tumour growth, spheroids could be grown in vitro in liquids of different viscosities (since the viscosity of the extracellular water depends crucially on the viscosity of any external liquid source).

With the short-range, attractive force switched on, the tumour evolved to a steady state (with the particular set of parameters chosen) in which cellular motion was always inward. In such a situation, there was no mass flux across the free surface, and the mass required for the generation of new cells came completely from recycling old cells. No area of necrosis was generated in this case.

We analysed the model for the special case in which the tumour was rich in oxygen and the cells proliferated at their maximal, oxygen-independent rate. We expect that this case is relevant during the early stages of avascular growth, whilst the tumour is still small enough that all cells are well-nourished. With suitable choices for the parameters, we were able to reduce the model to a quasi-steady limit. We found that the volume fraction of tumour cells was highest in the centre of the tumour, which we attributed to proliferation and the inability of the cells near the centre to migrate. Further, increasing the drag coefficient decreased the speed of tumour growth, as did increasing the viscosity. We also saw that increasing the viscosity increased the cell volume fraction at the boundary, and throughout the tumour, for a given width. In the limit of large viscosity, we found that the tumour cells filled space, and that there was no growth in this case because there was no extracellular water available to provide the building blocks for proliferation. The results of varying the key parameters in the oxygen-rich model agree well with the results of the numerical solution of the full model, even though they are a valid approximation only when the tumour remains small.

In developing our two-phase model, we have employed the simplest forms for each of the mechanisms included. We could modify these relations to make them more realistic. For example, we could use the 
Carman-Kozeny relationship for the permeability (see [14]). This is an empirically determined relationship that is used to describe the drag between water and sand grains that are nearly spherical, and captures the effect of the pore configuration. In this case,

$$
f_{c}=p_{w} \frac{\partial \alpha}{\partial x}+\frac{k_{1} \alpha^{2}}{(1-\alpha)^{2}}\left(u_{w}-u_{c}\right)
$$

and the equation replacing (21) would read

$$
\gamma \frac{\partial}{\partial x}\left(\alpha \frac{\alpha-\alpha^{*}}{\left((1-\alpha)^{2}\right.} H\left(\alpha-\alpha_{m i n}\right)\right)+\frac{k_{1} \alpha^{2} u_{c}}{(1-\alpha)^{4}}=2 \mu_{c} \frac{\partial}{\partial x}\left(\alpha \frac{\partial u_{c}}{\partial x}\right) .
$$

Our relationship for the cell-cell interaction term $\Sigma_{c}$ could also be modified to remove the jump in the pressure at $\alpha=\alpha_{\text {min }}$. To do this, we would consider a $\Sigma_{c}$ of the form

$$
\Sigma_{c}=\left\{\begin{array}{clc}
\gamma \frac{\left(\alpha-\alpha^{*}\right)\left(\alpha-\alpha_{\min }\right)}{(1-\alpha)^{2}} & \text { if } & \alpha>\alpha_{\min } \\
0 & \text { if } & \alpha<\alpha_{\min }
\end{array}\right.
$$

These and other modifications to the functional forms that are used to describe the various phenomena in our model still result in a second-order equation for the cell velocity of the form

$$
\frac{\partial}{\partial x}\left(f_{1}(\alpha) \frac{\partial u_{c}}{\partial x}\right)=f_{2}(\alpha) u_{c}+f_{3}(\alpha)
$$

In addition to providing a more physically-based description of avascular tumour growth, the model presented in this paper is a first step towards achieving a realistic model of vascular tumour growth. In order to do this, we plan to extend the existing model to include three phases: cells, extracellular water, and blood vessels. The modelling approach will follow directly from that presented herein; we merely require a further constitutive law to close the system. Such a model will be valuable to both experimentalists and clinicians for describing both the growth of vascular tumours in vivo and also for investigating the impact of therapies, including those that target the tumour's vasculature.

Three phase models could also be used to provide more detailed descriptions of other aspects of solid tumour growth. For example, we could include the effects of macrophages, extracellular matrix or a second population of tumour cells. Further, the multiphase modelling approach described in this paper could be used to describe a variety of other interesting and biologically important situations in which mass changes phase, such as modelling the growth of tissue engineered cartilage, bone remodelling, and soft tissue growth (see [1, 8,27 for examples of work that could be extended). In each case, the challenge of the modelling will be to capture accurately the stress relationships observed physiologically, and to incorporate correct representations of the sources and sinks for both mass and momentum. We believe that these models represent the natural modelling framework for mathematical study in these areas.

Acknowledgements. We wish to acknowledge many helpful discussions with Dr P. D. Howell. This work was carried out with the financial support of an EPSRC Ropa award (to CEL/HMB for CJWB) and an EPSRC Advanced Fellowship (HMB).

\section{References}

1. Barochas, V. H. \& Tranquillo, R.T. (1997) An anisotropic biphasic theory of tissue-equivalent mechanics: the interplay among cell traction, fibrillar network deformation, fibril alignment and cell contact guidance. $J$. Biomed. Eng., 119, 137-145.

2. Breward, C. J. W., Byrne, H. M. \& Lewis, C. E. (2001) Modelling the interactions between tumour cells and a blood vessel in microenvironment within a vascular tumour. Euro. J. Appl. Math.., 12, 529-556.

3. Breward, C.J.W. ET AL. (2001) Pericellular oxygen tension in experimental cell culture apparatus (working title). In Preparation.

4. Byrne, H. M. \& Chaplain, M. A. J. (1995) Growth of nonnecrotic tumours in the presence and absence of inhibitors. Math. Biosci, 2, 151-181.

5. Byrne, H. M. \& Chaplain, M. A. J. (1997) Free boundary value problems associated with the growth and development of multicell spheroids. Euro. J. Appl. Math., 8, 639-658. 
6. Byrne, H.M., King, J.R., McElwain, D.L.S. \& Preziosi, L. (2001) A two-phase model of solid tumour growth. Appl. Math. Lett.. In Press.

7. Byrne, H.M. \& Preziosi, L. (2001) Modelling solid tumour growth using the theory of mixtures. IMA J. Math. Appl. Med. Biol.. Submitted.

8. Cowin, S. (2000) How is a tissue built? J. Biomech. Eng., 122, 553-569.

9. Curtis, A. S. G. \& Seehar, G.M. (1971) The control of cell division by tension or diffusion. Nature, 274, $52-53$.

10. De Angelis, E. \& Preziozi, L. (2000) Advection-diffusion models for solid tumour evolution in vivo and related free boundary problems. Math. Mod. Meth. App. Sci., 10:(3), 379-407.

11. Dixon, M. \& Webb, E.C. (1979) Enzymes. Academic Press, New York.

12. Fitt, A. D., Howell, P. D., King, J. R., Please, C. P. \& Schwendeman, D.W. Poroelastic multiphase flow modelling for paper squeezing. Euro. J. Appl. Math. To appear.

13. Franks, S. J. (2001) Mathematical modelling of tumour growth and stability. PhD Thesis. University of Nottingham.

14. Fowler, A. C. (1997) Mathematical models in the applied sciences. Cambridge University Press, Cambridge.

15. Gatenby, R.A. \& Gawlinski, E.T. (1996) A reaction-diffusion model of cancer invasion. Cancer Res., 56, $5745-5753$.

16. Greenspan, H.P. (1972) Models for the growth of a solid tumour by diffusion. Stud. Appl. Math., 52, 317-340.

17. Greenspan, H. P. (1976) On the growth and stability of cell cultures and solid tumours. J. Theor. Biol., 56, 229-242.

18. Hahnfeldt, P., Panigraphy, D., Folkman, J. \& Hlatky, L. (1999) Tumour development under angiogenic signalling: a dynamic theory of tumour growth, treatment ersponse and postvascular dormancy. Cancer Res., 59, 4770-4775.

19. Helmlinger, G., Netti, P. A., Lichtenbeld, H. C., Melder, R. J., \& Jain, R. K. Solid stress inhibits the growth of multicellular tumour spheroids. Nature Biotech., 15, 778-783.

20. Hockel, M., Schlenger, K., Hockel S. \& Vaupel, P. (1999) Hypoxic cervical cancers with low apoptotic index are highly agressive. Cancer Res., 59(18), 4525-4528.

21. Krogh, A. (1919) The number and distribution of capillaries in muscles with calculations of the oxygen pressure head necessary for supplying the tissue. J. Physiol., 52, 409-415.

22. Landman, K. A. \& Please, C. P. (2001) Tumour dynamics and necrosis: surface tension and stability. IMA. J. Math. Appl. Med., 18:2, 131-158.

23. Pignatelli, M. (1998) Integrins, cadherins and catenins: molecular cross-talk in cancer cells. J. Pathol., 186:(1), $1-2$.

24. Santini, M.T. \& Rainaldi, G. (1999) Three-dimensional spheroid model in tumour biology. Pathobiology, 67:(3), 148-157.

25. Sherratt, J.A. (1993) Cellular growth and travelling waves of cancer. SIAM Appl. Math., 53, 1713-1730.

26. Sherratt, J.A. (2000) Wave front propagation in a competition equation with a new motility term modelling contact inhibition between cell populations. Proc. Roy. Soc Lond. A, 456, 2365-2386.

27. Simon, B. R., Liable, J. P., Pflaster, D., Yuan, Y., \& Krag, M. H. (1996) A poroelastic finite element formulation including transport and swelling in soft tissue structures. J. Biomed. Eng., 118, 1-9.

28. Ward, J. P. \& King, J. R. (1997) Mathematical modelling of avascular-tumour growth. IMA. J. Math. Appl. Med., 14, 39-69.

29. WARD, J. P. \& King, J. R. (1999) Mathematical modelling of avascular-tumour growth II: modelling growth saturation. IMA. J. Math. Appl. Med., 16, 171-211.

30. Wolff, M., Fandrey, J., \& Jelkmann, W. (1993) Microelectrode measurements of pericellular $\mathrm{pO}_{2}$ in erythropoietin-producing human hepatoma cell cultures. Am. J. Physiol, 265:(5), 1266-1270 


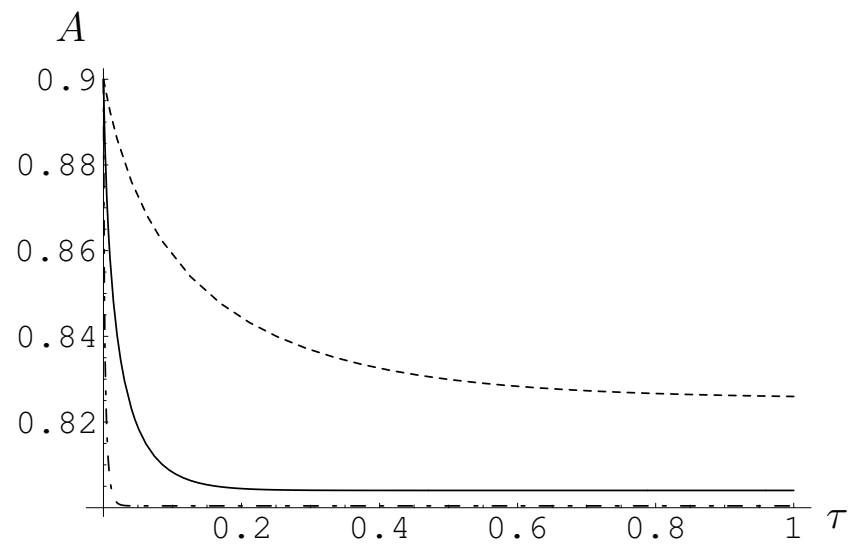

Fig. 1. Graph showing how $A$ varies with $\tau$ when $\mu=0.1$ (dot-dashed line), $\mu=1$ (Solid line), and $\mu=10$ (dashed line). Parameter values: $\alpha_{\min }=0.7, \alpha^{*}=0.8$ and $s_{5}=1 / 11$. 


\section{$A_{s}$}

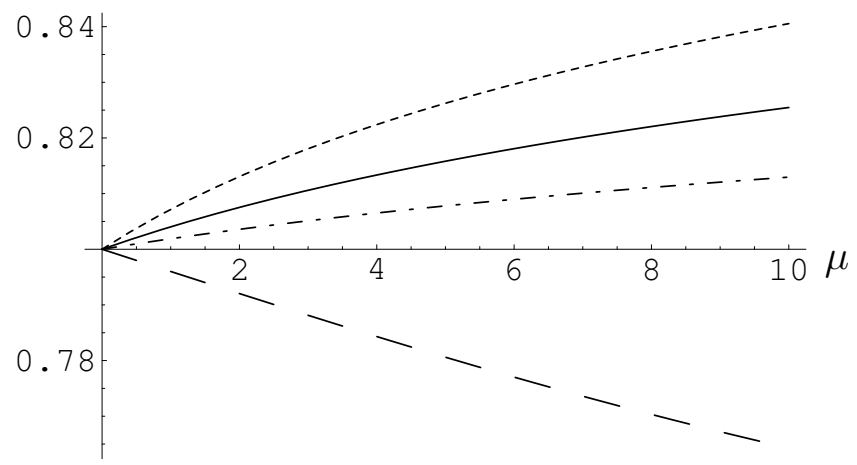

Fig. 2. Graph showing how $A_{s}$ varies with $\mu$, for $s_{5}=0$ (small dashed line), $s_{5}=1 / 11$ (solid line), $s_{5}=0.15$, (dot-dashed line) and $s_{5}=0.3$ (large dashed line). Parameter values: $\alpha_{\min }=0.7, \alpha^{*}=0.8$. 

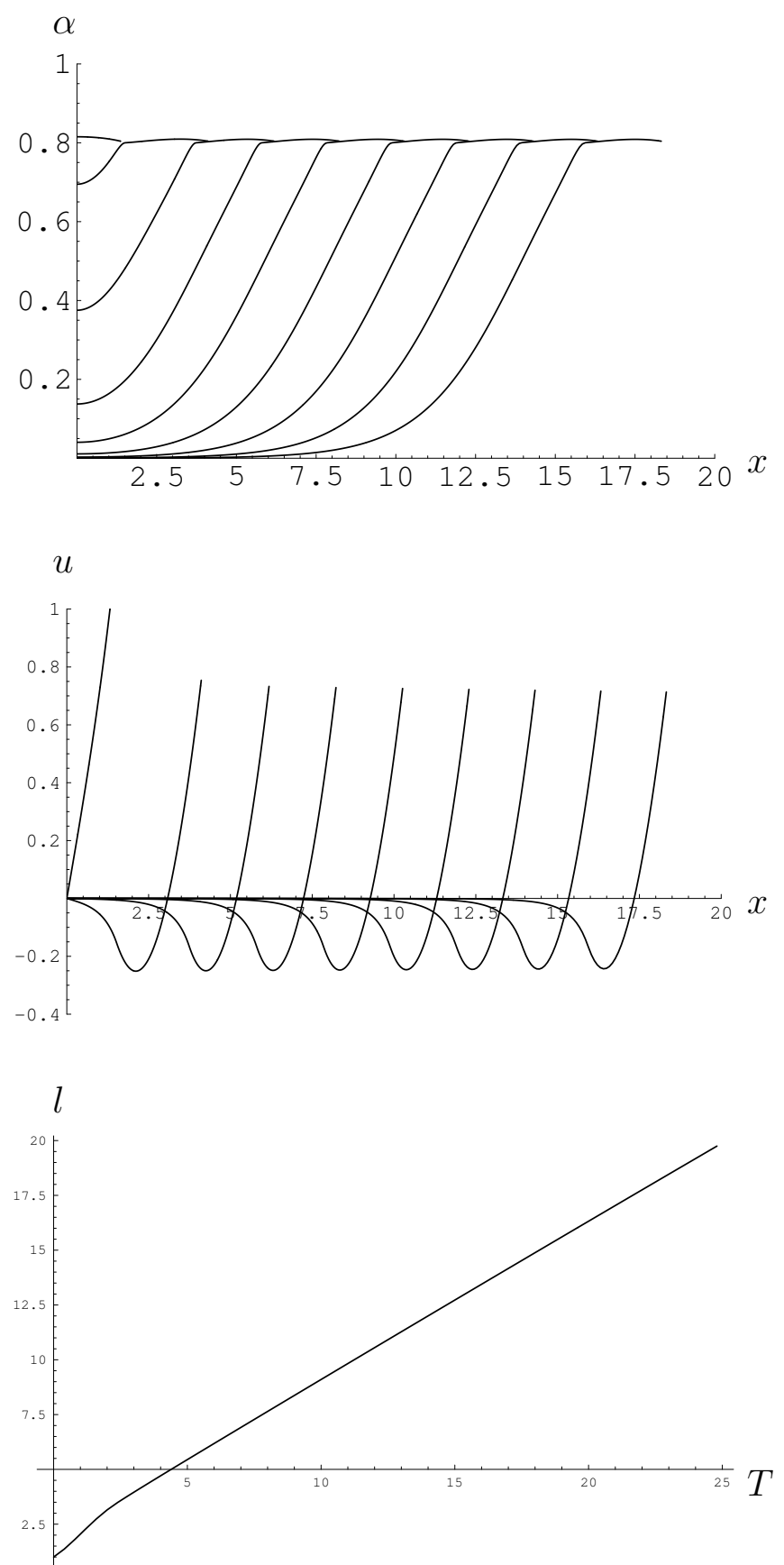

Fig. 3. Graphs showing (a) how $\alpha$ varies with $x=\xi l(T)$, for various values of $T(T=0.4,3.2,6,8.8,11.6,14.4$, 17.2, 20 and 22.8) (b) how $u$ varies with $x$, for various values of $T$, and (c) how $l$ evolves with $T$. 


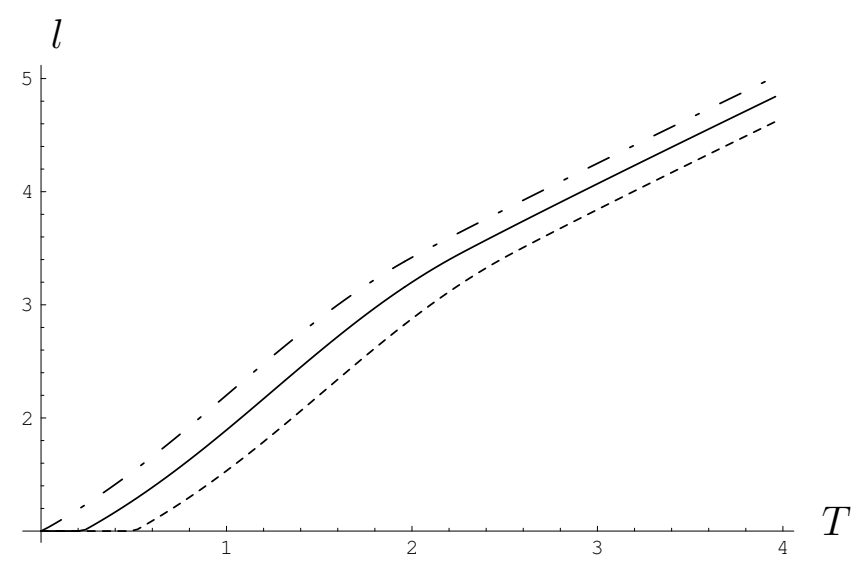

Fig. 4. Graph showing how $l$ varies with $T$, for $\alpha_{0}=0.1$ (dashed line) $\alpha_{0}=0.5$ (solid line) and $\alpha_{0}=0.8$ (dot-dashed line line). Parameter values: $\mu=0.25$ and $k=0.25$. 


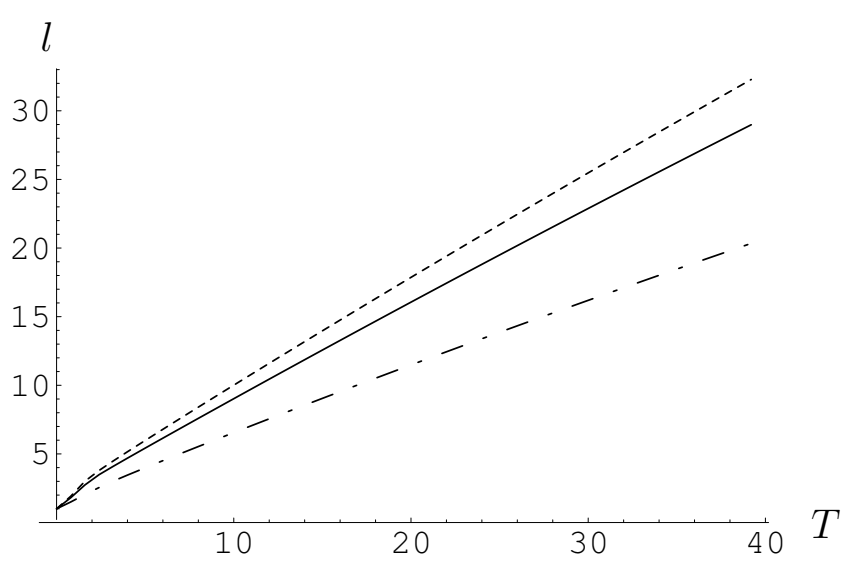

Fig. 5. Graph showing how $l$ varies with $T$, for $k=0.1$ (dashed line) $k=1.0$ (solid line) and $k=10.0$ (dot-dashed line). Parameter values: $\mu=1$. 


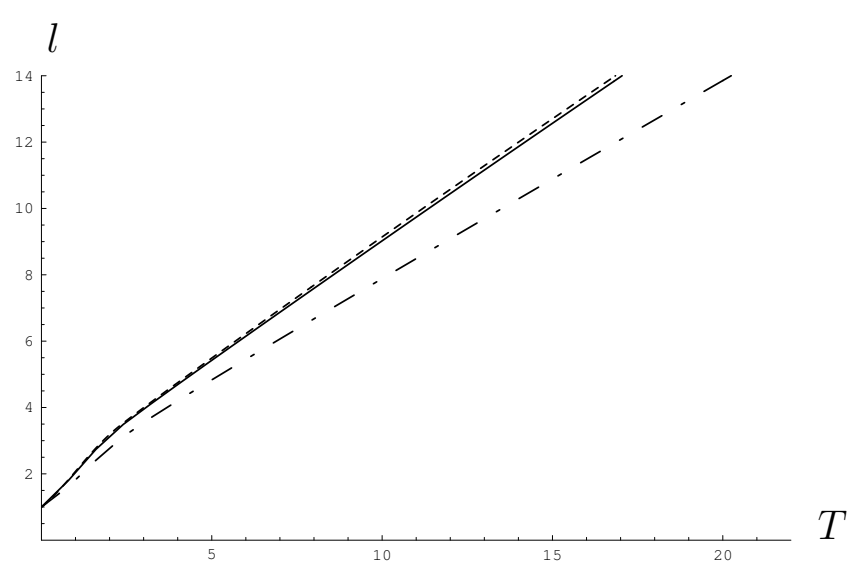

Fig. 6. Graph showing how $l$ varies with $T$, for $\mu=0.25$ (dashed line) $\mu=1.00$ (solid line) and $\mu=10.00$ (dot-dashed line). Parameter values: $k=1$. 


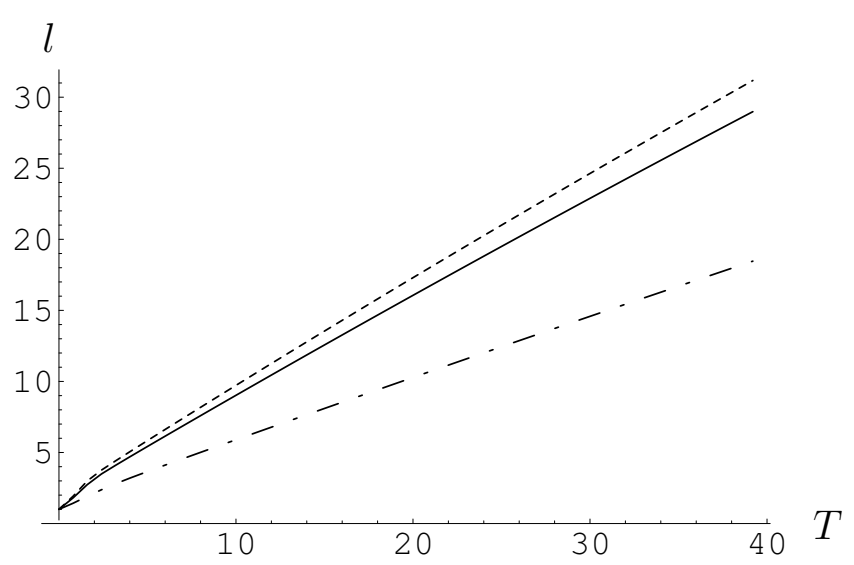

Fig. 7. Graph showing how $l$ varies with $T$, for $\mu=k=0.25$ (dashed line) $\mu=k=1.00$ (solid line) and $\mu=k=10.00$ (dot-dashed line). 
$\alpha$

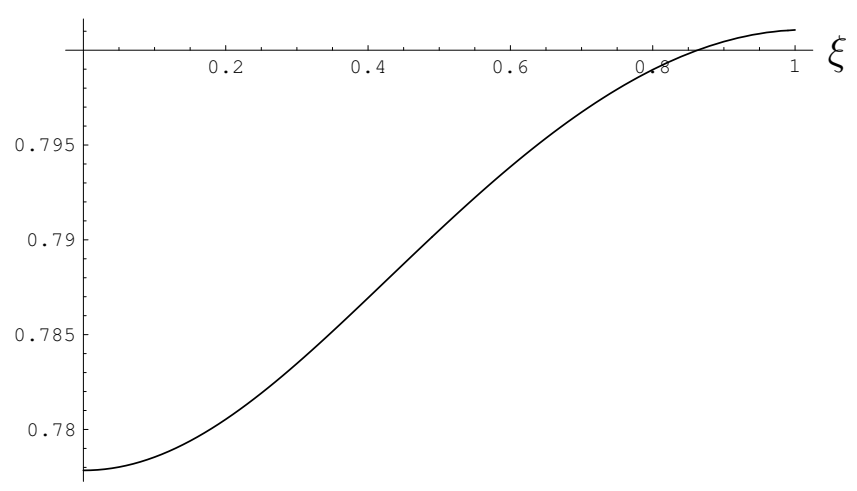

$u$
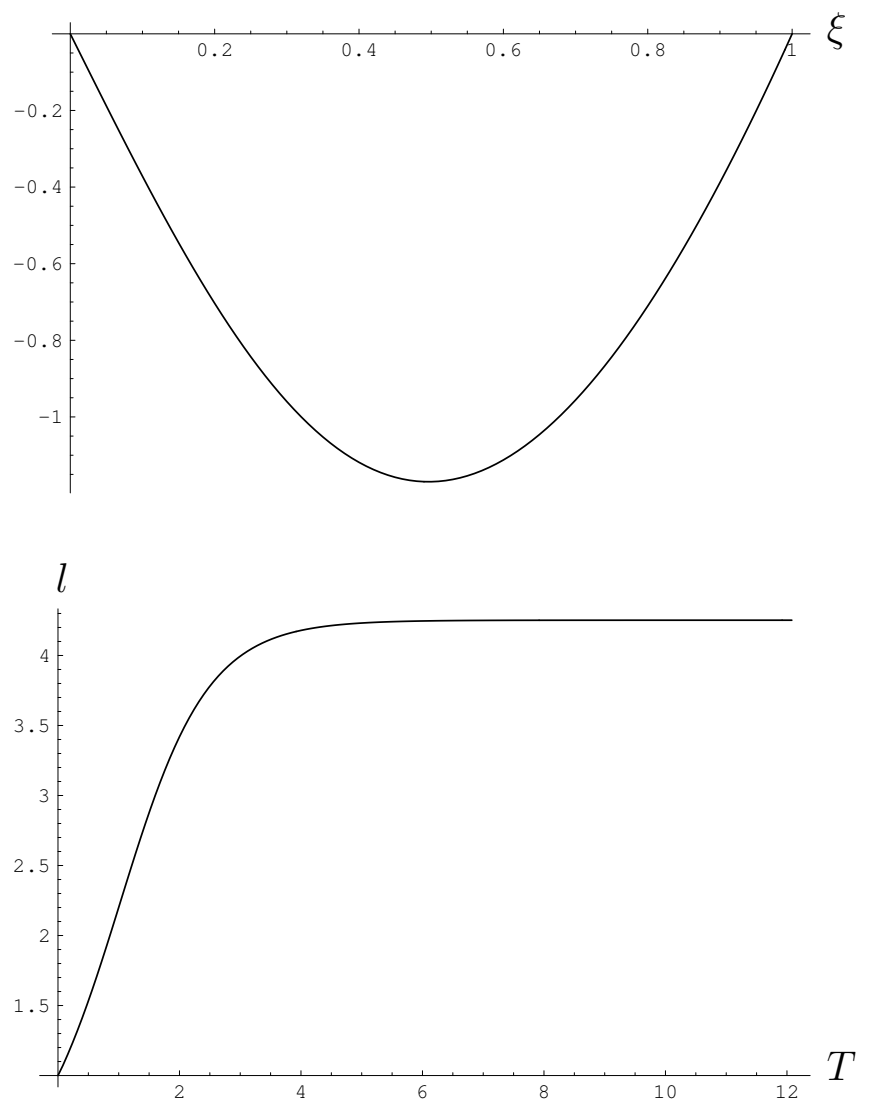

Fig. 8. Graphs showing (a) how $\alpha$ varies with $\xi=x / l$ and (b) how $u$ varies with $\xi=x / l$, in the steady state, and (c) how $l$ varies with $T$. Parameter values: $\mu=0.25, k=0.25, \alpha^{*}=0.8$, and $\alpha_{\text {min }}=0.6$. 


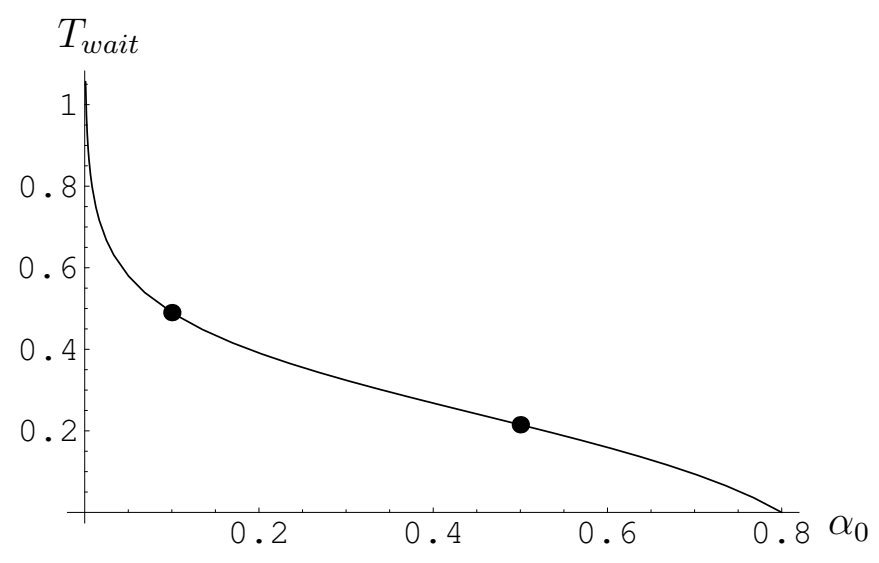

Fig. 9. Graph showing how the waiting time $T_{\text {wait }}$ varies with $\alpha_{0}$. The dots indicate the waiting times for tumours seeded with $\alpha_{0}=0.1$ and 0.5 (c.f. Figure 4). Parameter values $s_{5}=1 / 11, \alpha^{*}=\alpha_{\text {min }}=0.8$ 


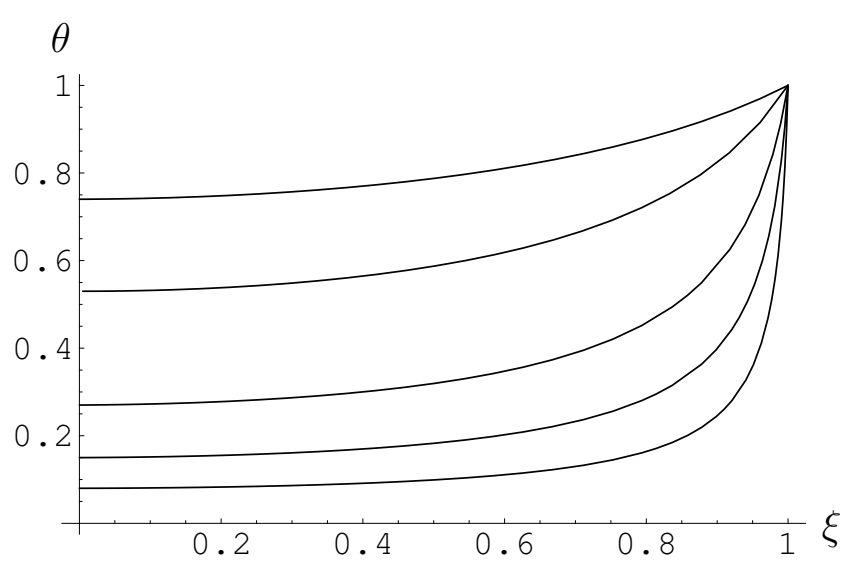

Fig. 10. Graph showing how $\theta$ varies with $\xi=x / l$, for $l=1$ (highest line), 2, 5, 10 and 20 (lowest line). 


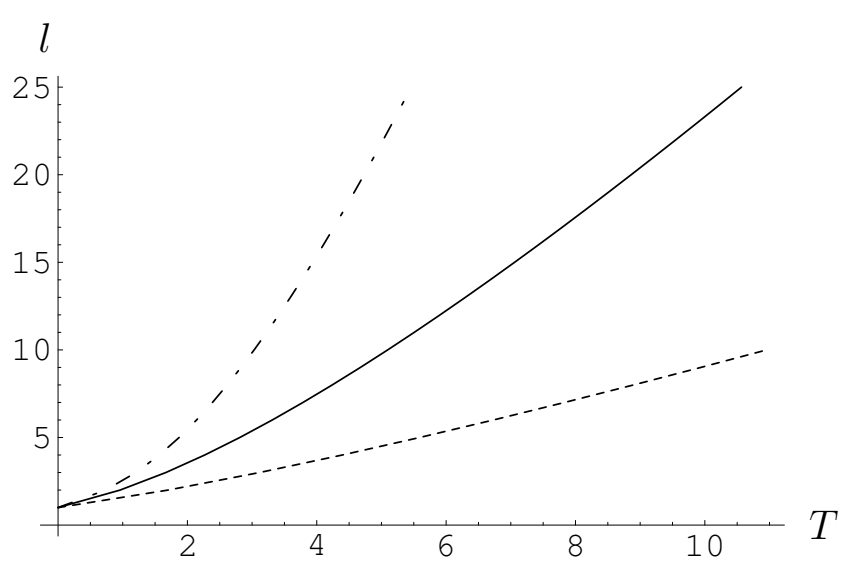

Fig. 11. Graph showing how $l$ varies with $T$, for $\kappa=0.1$ (dashed line), 1 (solid line), and 10 (dot-dashed line). 


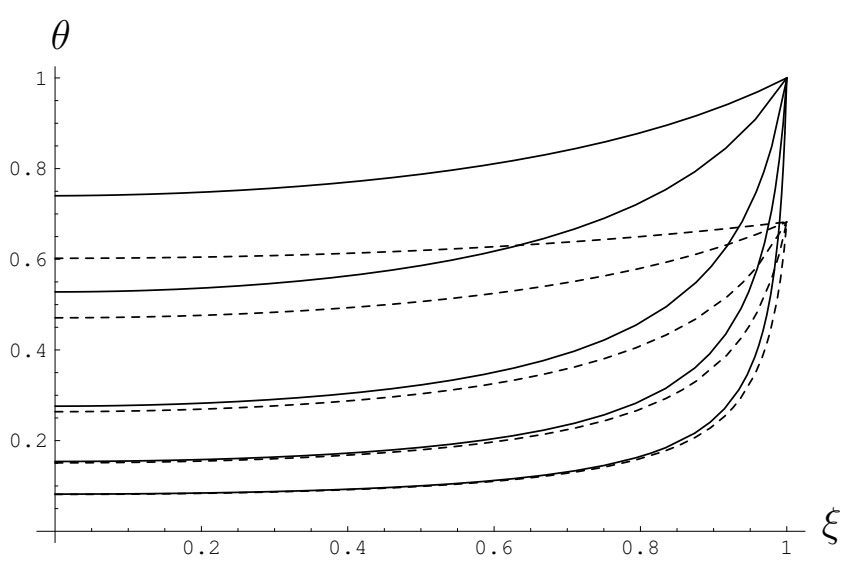

Fig. 12. Graph showing how $\theta$ varies with $\xi=x / l$, for $\nu=0$ (solid line) and $\nu=1$ (dashed line) for tumours of width $l=1$ (highest lines), 2, 5, 10 and 20 (lowest lines). 


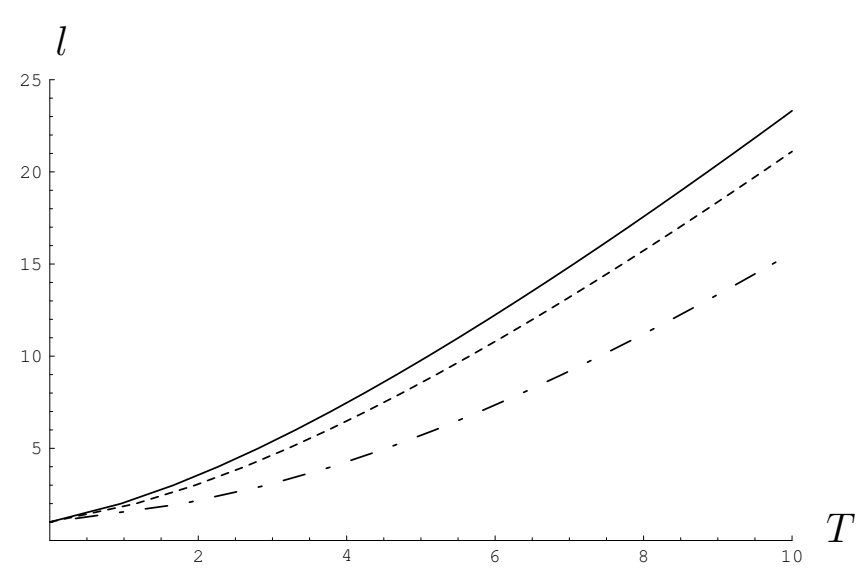

Fig. 13. Graph showing $l$ against $T$ for $\nu=0$ (dashed line), $\nu=1$ (solid line) and $\nu=10$ (dot-dashed line). 